\title{
El tatuaje y la perforación en la construcción de la corporeidad
}

\author{
Tattoo and piercing as elements of corporal building
}

Tatuagem e piercing na construçao do corporal

\author{
Alberto González García \\ Doctor en Historia de la Enfermería. Facultad de Enfermería de Cuenca. Universidad de Castilla-La Mancha. \\ Cómo citar este artículo en edición digital: González García, A. (2013) El tatuaje y la perforación en la construcción de la \\ corporeidad. Cultura de los Cuidados.17, 37. Disponible en: <http://dx.doi.org/10.7184/cuid.2013.37.03> \\ Correspondencia: Alberto González García- Facultad de Enfermería de Cuenca. Universidad de Castilla-La Mancha. Camino \\ del Pozuelo s/n, 16071 Cuenca. Tlfno. 969.17.91.00, extensiones 4641, 4640 \\ Correo electrónico: alberto.gonzalez@uclm.es
}

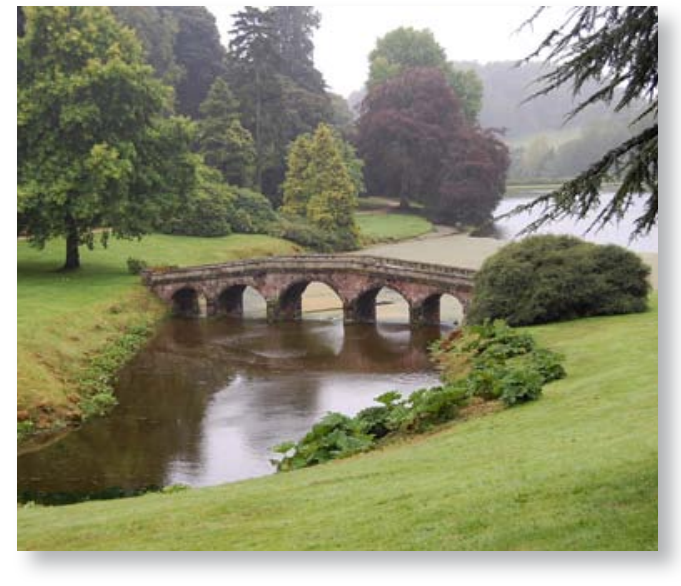

ABSTRACT

Tattooing and body piercing are a reflection of the human need to decorate their body. Young people create significant senses that create other identities. The target of this research is to reveal the meaning of the tattooed skin to understand this social phenomenon. We understand these practices as a network of meanings, expressed in attitudes and feelings, which is crossed by the gender, the experience of pleasure VS pain and resistance to the dominant aesthetic. We have selected a sample of six men and six women of all clients served in 2009 on a tattoo and piercing Clinic to conduct in-depth interviews. There is a difference between feminine and masculine, both in de- sign and corporal areas such as the decision leading to tattooing or piercing, and attributed meaning. Women choose these body practices with an aim of playing and seduction, they feel under the gaze of society, like men, but men do not feel as strongly beauty standards than women. Customers feel discrimination in the family and work, and they explain that tattoos and piercings are not valued as an artistic element in society.

Key words: body, body image, tattoo, piercing.

\section{RESUMO}

Tatuagem e piercing são um reflexo da necessidade humana para decorar o seu corpo. Os jovens criam significativa de sentidos diferentes que contribuem a criar outras identidades. $\mathrm{O}$ objetivo desta pesquisa é revelar o significado da pele tatuada para entender este fenômeno social. Compreendor-nos essas práticas como uma rede de significados, expressos em atitudes e sentimentos, que é atravessado pelo gênero, a experiência de prazer-dor e resistência à estética dominante. Foi selecionada uma amostra de seis homens e seis mulheres de todos os clientes atendidos em 2009 em uma clínica de tatuagem e piercing para con- 
duzir entrevistas em profundidade. Há uma diferença entre feminino e masculino, tanto em design e áreas perturbadas como a decisão que leva à tatuagem e piercing e atribuiu significado. As mulheres escolhem essas práticas corporais com o objetivo de reprodução e sedução, sentem-se sob o olhar da sociedade, na mesma que os homens, mas eles não sentem tão fortemente os padrões de beleza. Os usuários sentem a discriminação na família e no trabalho, e explicou que tatuagens e piercings não são valorizadas como um elemento artístico na sociedade.

Palavras-chave: corpo, imagem corporal, tatuagem, piercing.

\section{RESUMEN}

El tatuaje y la perforación corporal son un reflejo de la necesidad del ser humano por decorar su cuerpo. Los jóvenes crean significantes de sentidos diferentes que contribuyen a crear otras identidades. El objetivo de esta investigación es desvelar los significados bajo la piel tatuada para comprender este fenómeno social. Entendemos estas prácticas como un entramado de significados, expresados en actitudes y sentimientos, que se encuentra cruzado por el género, la experiencia del placer-dolor y la resistencia ante las estéticas dominantes. Hemos seleccionado una muestra de seis hombres y seis mujeres del total de usuarios atendidos durante el año 2009 en una Clínica de tatuaje y perforación, para realizar entrevistas en profundidad. Existe una diferenciación entre lo femenino y lo masculino, tanto en los diseños y zonas alteradas, como en la decisión que lleva al tatuaje y perforación y significado atribuido. Las mujeres eligen estas prácticas corporales con un objetivo de reproducción y seducción, se sienten bajo la mirada de la sociedad, igual que los hombres, pero ellos no sienten con la misma fuerza las normas de belleza. Los usuarios sienten discriminación en los ámbitos familiar y laboral, y explican que los tatuajes y las perforaciones no son valorados como elemento artístico por la sociedad.

Palabras clave: cuerpo, imagen corporal, tatuaje, perforación.

\section{INTRODUCCIÓN}

El tatuaje y la perforación corporal son dos de las prácticas más antiguas y comunes en las diferentes culturas, y son un reflejo de la necesidad del ser humano por decorar su cuerpo. Las técnicas nacieron desde cada cultura, que en situaciones diferentes buscaban su propia manera de expresarse (López Vergara, 2007).

Los tatuajes más arcaicos encontrados son de un cuerpo de más de 5300 años encontrado en los Alpes italianos, o los encontrados en Egipto en momias de hace 4000 años (Tripaldi, 2004). También existen ejemplos en los indígenas americanos de la era precolombina (Pérez Cachafeiro et al, 2003; Pérez Cotapos y Cossío, 2006). Las primeras muestras de perforaciones datan del año 709 a. C. (Calero Martín de Villodres et al, 2010).

La persistencia del tatuaje y la perforación durante siglos muestra la fuerte necesidad del ser humano por marcar su cuerpo (Tripaldi, 2004). Esta necesidad de autorrepresentarse, de ser agradable exteriormente a su comunidad, contiene las múltiples significaciones del sujeto en su entorno social, de forma que permite una notoriedad, una diferenciación con el otro, diferente de sí mismo, pero similar a los demás sujetos que practican la misma experiencia y que comprenden los símbolos (López Vergara, 2007). En cualquier cultura el cuerpo está íntimamente ligado a lo social, y toda 
práctica social es una experiencia corporal simbólica y compartida (Esteban, 2004; Acuña Delgado, 2006).

Pero las formas concretas por las cuales las sociedades expresan su relación con lo corporal son diversas y variables, es decir, que lo que distingue a unas culturas de otras es el grado de diversidad y relevancia, en parámetros técnicos, estéticos y sociales, que asignen las mismas a esta práctica (Arcos y Galindo Huertas, 2010). Sólo un análisis histórico y transcultural nos permitiría obtener en toda su complejidad un panorama completo de significados y prácticas (Esteban, 2004; López Vergara, 2007).

La visión que tradicionalmente aborda la juventud la caracteriza como un momento transitorio, sin identidad, entre dos grupos reconocidos socialmente, la niñez y la adultez, en donde se produce una pérdida de los valores sociales. Con esta perspectiva, quedan en el olvido las profundas transformaciones $y$ los grandes cambios que ocurren en el mundo contemporáneo, los cuales se expresan en las culturas juveniles. Los procesos que articulan la cultura generacional con el cambio de época tienen su visibilidad social en la construcción social de lo joven. El valor que tiene lo joven y la experiencia de identidad vivida por los propios jóvenes los convierte en actores sociales que son un elemento identificatorio fundamental de la contemporaneidad. Nunca como ahora se ha identificado a la juventud con la permanente novedad que particulariza lo moderno; así lo joven se identifica con lo actual y adquiere el carácter de símbolo (Tripaldi, 2004; Beltrán Beltrán, 2012).

Los jóvenes de hoy forjan un nuevo mundo en la cotidianeidad de sus vidas, en su vestir y actuar, en sus consumos culturales, que está determinando el inicio de otra etapa en la historia cultural juvenil (Tripaldi, 2004). Los cuerpos de los jóvenes son un elemento generador de cultura e intención, reflejan aspectos económicos, sociales y políticos del entramado social en el que opera (Acuña, 2006). Los jóvenes están constantemente creando nuevos significantes (vestuario, peinado, lugares, etc.) que poseen sentidos diferentes y que a su vez contribuyen a crear otras identidades. Precisamente estas identidades hablan del presente y de cómo los jóvenes se apropian de este momento de la historia y cómo en su vida cotidiana van construyendo el futuro. Hoy en día, por tanto, se produce una re-significación de los espacios, como espacios culturales y simbólicos, que se convierte en espacio de identificación y de expresión (García Hernández, 2004; Tripaldi, 2004).

El objetivo de esta investigación es desvelar los significados bajo la piel tatuada, los cuales nos pueden guiar en cierta forma a comprender este fenómeno social. Entendemos las prácticas del tatuaje y la perforación como un entramado de significados, expresados en actitudes y sentimientos a nivel personal/ psicológico y a nivel social/comunitario. Tal entramado se encuentra cruzado por diversas categorías, entre ellas, el género, la clase social, la religión, la valoración y experiencia del placer-dolor, y la resistencia ante las estéticas dominantes (Esteban, 2004; Ortiz Gómez, 2006; López Vergara, 2007). Centrándonos en alguna de estas categorías mencionadas, nuestra hipótesis de partida es valorar hasta qué punto existen diferencias de género en la motivación de las personas a la hora de tatuar y perforar sus cuerpos. Es decir, hasta qué punto estos comportamientos que forman parte de la identidad son diferentes o similares en cada género y cómo cada grupo concibe y valora su propio cuerpo de diferente forma. 


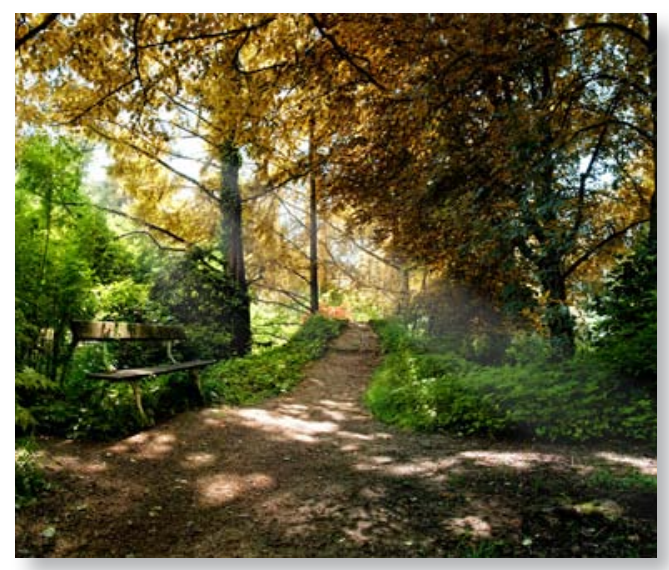

\section{METODOLOGÍA}

Este trabajo forma parte de otro proyecto más amplio en el que se pretende conocer la actividad asistencial de una Clínica de tatuaje y perforación de la ciudad de Granada (España) durante el año 2009. Por un lado, se han extraído los datos globales de la actividad asistencial de la clínica para realizar una presentación detallada que relacione los ítems de edad, sexo, zona anatómica y fecha de tatuaje o perforación. En este caso, los datos han sido codificados ajustándonos a la Ley Orgánica 15/1999, de 13 de diciembre, de Protección de Datos de Carácter Personal.

Por otro lado, se realizaron un total de doce entrevistas en profundidad a personas que fueron tatuadas, en concreto seis mujeres y seis varones. En este caso, sus edades oscilaron entre los 18 y los 30 años. En relación con el número de personas entrevistadas, se ha seguido el principio de parsimonia descrito por Campbell (1955) que establece que no por utilizar más informantes tiene por qué mejorar la calidad de la información. Esto es, que elegir un mayor número de informantes no tiene por qué ser mejor, sino que es preferible emplear menos informantes pero mejor cualificados.

La búsqueda y selección de los participantes en las entrevistas en profundidad se hizo de manera intencional, siguiendo los criterios de disposición a cooperar con el investigador, capacidad de comunicación e imparcialidad (Tremblay, 1957), conocimientos del informante sobre su propia cultura, que el informante no sea analítico al comunicar sus conocimientos (Spradley y McCurdy, 1972), y que se pueda hablar con él/ella fácilmente (Bernard, 2002).

Este trabajo, por tanto, utiliza tanto metodología cuantitativa, con un análisis estadístico descriptivo, como metodología cualitativa, con entrevistas en profundidad. Las entrevistas fueron no estructuradas, en las que el investigador se limitó a llevar al informante hacia un tema y se dejó que hablara (Álvarez Roldán, 2001). Según Gorden (1975), este tipo de entrevistas tienen mayor validez que las estructuradas, ya que se producen en una atmósfera más relajada que facilita la comunicación entre el informante y el entrevistador, son más flexibles, permitiendo adaptar las preguntas a cada tipo de informante, y hacen posible abordar temas relacionados con la experiencia inconsciente del informante.

Finalmente, se transcribió lo grabado, además de tomar notas sobre el contenido, las circunstancias y la actuación del informante durante la conversación.

\section{RESULTADOS}

La actividad asistencial media de la clínica estuvo por encima de las 150 perforaciones al mes durante el año 2009, con periodos de especial actividad durante los meses de julio (206 perforaciones), agosto (227), septiembre (189) y diciembre (200).

El perfil de usuario de la clínica es una mujer mayor de edad (casi la mitad de las usuarias), seguido de los varones mayores de edad (que representan un cuarto de la clientela). Si 
tenemos en cuenta todo el rango de edad, se observa una clara tendencia a la feminización de la clientela, ya que las mujeres suponen el $70 \%$ del total.

Las zonas anatómicas preferidas por las mujeres son el labio ( $26 \%$ de las perforaciones en mujeres), ombligo (19\%) y nariz (17\%), seguido de otras localizaciones (la más frecuente es el "surface" o perforación superficial encima del ombligo). Sin embargo, entre los varones son la ceja (22\% de las perforaciones en varones), el tragus (22\%), el labio (19\%) y el pezón (13\%) los más demandados. Estas pautas son muy similares a las encontradas en otros países europeos, si bien la frecuencia en la perforación de la lengua es inferior en nuestro país (Antoszewski et al, 2006; Bone et al, 2008; Gutsche et al, 2008; Cegolon et al, 2010). El piercing genital, pese a llamar la atención entre los jóvenes, es muy poco demandado (3\% entre los varones, $1 \%$ entre las mujeres).

Una presentación más detallada que relacione la distribución de los piercings por zona anatómica y fecha de realización sugiere pautas de comportamiento específicas. De este modo, puede observarse un aumento en la frecuencia de piercings en el ombligo entre las mujeres durante los meses de mayo, junio, julio y agosto, periodo veraniego, en donde puede lucirse con mayor facilidad.

En relación a los tatuajes, existe una clara $\mathrm{y}$ vigente diferenciación entre lo femenino $\mathrm{y}$ lo masculino, tanto en los diseños, en las zonas alteradas, como en la decisión que lleva al tatuaje y perforación y en el significado atribuido. Por ejemplo, los hombres prefieren lugares visibles, expuestos y de gran extensión, tales como los brazos y antebrazos, espalda, y pantorrillas. En cambio, las mujeres prefieren zonas vinculadas a la sensualidad y el erotismo, algo más cubiertas: tobillos, vientre, zona próxima a los senos, zona lumbar, hombros. Tal diferenciación se hace patente también en la elección del diseño. Mientras los hombres prefieren representaciones de símbolos expresamente relacionados al físico y a la demostración de virilidad (dragones, serpientes, armas), las mujeres eligen diseños más relacionados con la delicadeza: hadas, enredaderas, lunas, corazones, tribales.

Se seleccionaron un total de doce usuarios del total de personas que acudieron a la clínica para tatuarse el cuerpo para realizar las entrevistas en profundidad, seis hombres y seis mujeres. Según estos resultados, el mundo del tatuaje y de la perforación corporal continúa siendo visto hoy en día como negativo y prohibido. Los usuarios sienten una cierta discriminación por la sociedad en general, no así por su entorno de iguales, de lo cual son conscientes. Esta discriminación se ve reflejada en diversos ámbitos de sus vidas, fundamentalmente familiar y laboral (López Vergara, 2007). De este modo, generan un discurso que pueda responder ante tal segregación parcial. La explicación dada por los usuarios es que los tatuajes y las perforaciones no son valorados como elemento artístico por la sociedad, sino que estas prácticas están situadas lejos de los centros formales económicos, artísticos, higiénicos, comportamentales y políticos, perdiendo así su estatus. Aquí, es donde existe una clara visión adultocéntrica respecto a las alteraciones, dentro de la cual se develan discursos fuertemente ligados a las buenas costumbres y a los deberes de los jóvenes. Creemos que estos prejuicios están originados a partir de una concepción estereotipada del cuerpo, basados en la tradición judeocristiana del mismo utilizado sólo para fines prácticos, funcionales y reproductivos (Arcos y Galindo Huertas, 2010). Además, las marcas corpora- 
les son vistas como una flagelación y no como un deseo de permanencia. El cuerpo es visto simplemente como un accesorio, de tal forma que no es considerado como un objeto de experimentación e innovación por parte de las culturas juveniles (López Vergara, 2007).

\section{DISCUSIÓN Y CONCLUSIONES}

En Occidente, la alteración corporal siempre ha estado encaminada hacia la demostración simbólica de ciertas características, la mayoría de ellas vinculadas hacia lo masculino, la fortaleza, y la virilidad, las cuales han sido naturalizadas socialmente (Rigol Cuadra, 2006; López Vergara, 2007). Mujeres y hombres están influidos de manera distinta en cuanto a su cuerpo y apariencia, y eso se relaciona con la definición de lo femenino y lo masculino, donde la belleza sigue estando más asociada a lo femenino y la fuerza a lo masculino, con un tratamiento diferente de la sexualidad y el deseo para hombres y mujeres (Esteban, 2004; Beltrán Beltrán, 2012).

En general, pese a que la bibliografía existente insiste en que los hombres son enseñados en la exhibición e instrumentalización de su cuerpo para la fuerza y el trabajo, en donde prevalece un canon de belleza caracterizado por el vigor varonil (Esteban, 2004), los hombres de nuestro estudio muestran una tendencia a elegir estas prácticas corporales con un objetivo de seducción, muy similar al mostrado por las mujeres. Los objetivos principales del aprendizaje corporal de las mujeres son la reproducción y la seducción, habida cuenta de que se sienten bajo la mirada constante de la sociedad (Rigol Cuadra, 2006), al igual que los hombres, aunque estos últimos no sienten con la misma fuerza el efecto de las normas de belleza, la moda en el vestir, o la necesidad del arreglo constante.
Habitualmente, se considera la forma en que las mujeres cultivan su apariencia de un modo bastante restrictivo, como una subordinación a las normas dominantes y bajo la influencia directa de los discursos hegemónicos, como si las personas fueran seres pasivos sometidos a las leyes del mercado e industrias de la belleza. Creemos, sin embargo, que no se trata de un simple seguimiento de los discursos dominantes, sino que las mujeres y los hombres gestionan su imagen negociando al mismo tiempo su lugar en la sociedad. De este modo, las personas son reguladas desde fuera, pero ellas mismas buscan ser las protagonistas directas de ese control. El cuerpo se configura como un elemento que reivindicar, que gusta de ser exhibido, y para ello debe ser cuidado con esmero y que, por tanto, centra las actividades cotidianas. Se trata el cuerpo como símbolo de estatus, juventud, salud, energía y objeto de deseo (Esteban, 2004).

La regulación de lo corporal alcanza su perfección en nuestros días a través de un doble juego, de potenciación del consumo y del fomento del autocontrol. Consumo y control que se ejercen específicamente en relación a cuatro grandes áreas: alimentación, ejercicio físico y deporte, cuidado estético y sexualidad (Beltrán Beltrán, 2012). El cuerpo es puesto en relación con la construcción social del concepto de persona. Por tanto, ejerce un papel conformador de la subjetividad de las personas como seres individuales y sociales. En este marco, la imagen corporal y el cuerpo individual y social son fundamentales en la construcción de la propia identidad y pertenencia a los diferentes grupos (Esteban, 2004).

Para comprender el tatuaje y la perforación se debe concebir el cuerpo como un mapa de inscripciones identitarias y como un espacio de comunicación y estetización, el cual permi- 


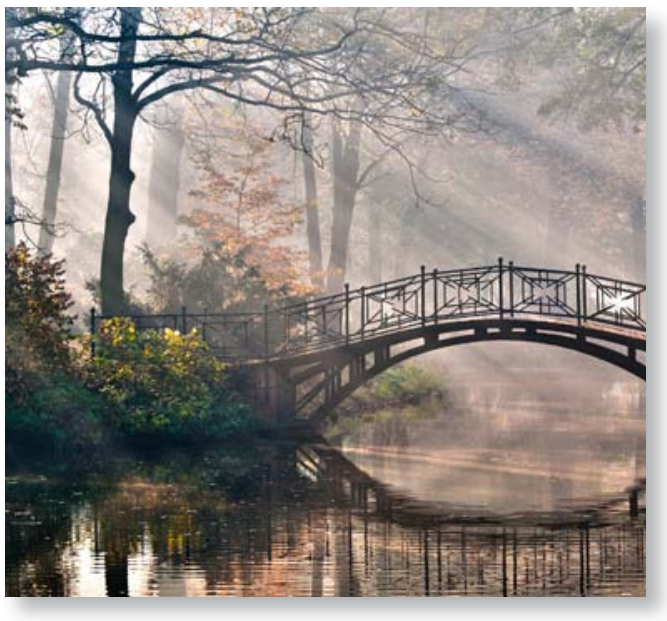

te encontrar sentidos que llevan a la identificación con uno mismo y con el grupo de iguales. El cuerpo es concebido como un cuerpo estético, y en él se invierte tiempo, esfuerzo y dinero para ser exhibido, en el sentido de anotar y hacerse notar, mirar y ser mirado. Los ornamentos del cuerpo adquieren así una estética corporal. De esta forma, el cuerpo es capaz de generar por un lado una conexión grupal, en tanto que la apariencia, los adornos, la forma de vestir, se vuelven un factor de inclusión; o un elemento de exclusión social, que no considera esta forma de representación dentro de los patrones formales de comportamiento de la juventud.

Se nos presenta una gama de significados corporales, el cuerpo como superficie de inscripción, representación o mapa de la condición social, la pertenencia al grupo de iguales, la manifestación del yo; como lugar de construcción del género; como lugar de conocimiento y poder, lugar de resistencia y reinscripción, transformable, controlable desde sí mismo; como sistema de clasificación social y simbólica, como emblema; como lugar de experiencia, memoria y subjetividad (Ortiz Gómez, 2006). Las prácticas corporales son contextuales, es decir, tienen que ver con la cultura, la clase, la etnia, el lugar, el tiempo, y el género.

El cuerpo, en definitiva, es así entendido como el lugar de la vivencia, el deseo, la reflexión, la resistencia, la contestación y el cambio social, en una interacción compleja con las dimensiones económicas, políticas, sexuales, estéticas e intelectuales.

\section{BIBLIOGRAFÍA}

- Acuña Delgado, A. (2006) Etnología de la danza rarámuri en la Sierra Tarahumara. Universidad de Granada, Granada.

- Álvarez Roldán, A. (2001) Entrevista semiestructurada sobre conductas de riesgo a adolescentes y jóvenes. $\mathrm{Mi}$ nisterio de Ciencia y Tecnología, Granada.

- Antoszewski, B.; Sitek, A.; Jędrzejczak, M.; Kasielska, A.; Kruk-Jeromin, J. (2006) Are body piercing and tattooing safe fashions? European Journal of Dermatology 16 (5): 572-575.

- Arcos, F.; Galindo Huertas, S. (2010) La exposición corporal y el cuidado de enfermería. Cultura de los Cuidados 28: 63-70.

- Beltrán Beltrán, C. (2012) La influencia de la cultura occidental en los cuidados del cuerpo relatives a la estética, a la actividad física y a la alimentación. Cultura de los Cuidados 34: 11-19.

- Bernard, H.R. (2002) Research methods in Anthropology: qualitative and quantitative methods. Walnut Creek, AltaMira Press.

- Bone, A; Nichols, T; Noah, N.D. (2008) Body piercing in England: a survey of piercing at sites other than earlobe. BMJ 336: 1426-1433.

- Calero Martín de Villodres, P; García Castillo, N; Basso Ruiz, C. (2010) Perforaciones cutáneas: cuidados y riesgos de complicaciones desde el punto de vista de enfermería. Ediciones Sider, Granada.

- Campbell, D.T. (1955) The informant in quantitative re $\neg$ search. The American Journal of Sociology 60: 339342.

- Cegolon, L; Miatto, E; Bortolotto, M; Benetton, M; Ma- 
zzoleni, F; Mastrangelo, G. (2010) Body piercing and tattoo: awareness of health related risks among 4,277 Italian secondary school adolescents. Public Health 10: 73-91.

- Esteban, M.L. (2004) Antropología del cuerpo. Género, itinerarios corporales, identidad y cambio. Bellaterra, Barcelona.

- García Hernández, A.M. (2004) El cuerpo como práctica artística y primer instrumento de los cuidados. Cultura de los Cuidados 15: 26-31.

- Gorden, R.L. (1975) Interviewing: strategy, techniques and tactics. Dorsey Press, Homewood.

- Gutsche, P; Schmalz, G; Landthaler, M. (2008) Prevalence of piercing in a German population. European Journal of Dermatology 18 (1): 26-28.

- López Vergara, R. (2007) Cuerpos transgresores/cuerpos transgredidos. Carne y memoria marcadas. Los jóvenes y sus prácticas de modificación corporal. Última Década 26: 103-119.

- Ortiz Gómez, T. (2006) Medicina, historia y género. 130 años de investigación feminista. KRK ediciones, Oviedo.
- Pérez Cachafeiro, S; Atitar de la Fuente, A; Díez Pérez, M.D; Montero Vacas, N. (2003) Perforaciones inconscientes. Breve descripción del fenómeno del piercing y sus posibles complicaciones. Atención Primaria 32 (9): 535-540

- Pérez-Cotapos, M.L; Cossío, M.L. (2006) Tatuajes y perforaciones en adolescentes. Revista Médica de Chile 134: 1322-1329.

- Rigol Cuadra, A. (2006) El otro cuerpo de la identidad: análisis de los modelos culturales de los trastornos del cuerpo femenino. Cultura de los Cuidados 19: 47-54.

- Spradley, J.P; McCurdy D.W. (1972) The Cultural Experience: Ethnography in Complex Society. Waveland Press, Long Grove.

- Tremblay, M.A. (1957) The key informant technique: a nonethnographic application. American Anthropologist 59: 699-701.

- Tripaldi, A.M. (2004) Identidades juveniles. Tatuaje y piercing en Cuenca. Universidad de Azuay, Cuenca (Ecuador)

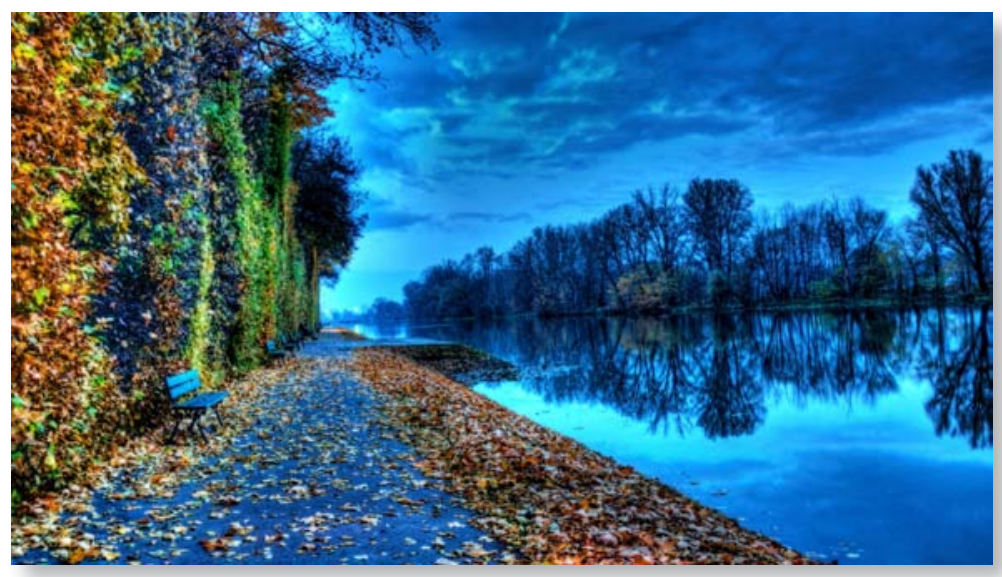

